\title{
Buddy-Motivational Interviewing (buddy-MI) to Increase Physical Activity in Community Settings Study Protocol for a Pragmatic Randomised Controlled Trial
}

\author{
David Brinson, MHealSc ${ }^{1}$, Mark Wallace-Bell, $\mathrm{PhD}^{1}$, Ray Kirk, $\mathrm{PhD}^{1}$, Andrew Hornblow, $\mathrm{PhD}^{1}$
}

\begin{abstract}
This article describes the development and evaluation of a novel buddy-motivational interviewing intervention intended to help apparently healthy but relatively sedentary adults to adopt and maintain regular physical activity for health and fitness. Many people experience great difficulty in initiating physical activity ("the getting going problem") and behavioural regression is common ("the keeping it going problem"). Typically there is a rather large gap between what people know to be healthy and what they actually do. This intervention is an adaptation of motivational interviewing in that it adds client-selected motivational-buddies who can provide in-session input as well as ongoing out-of-session support focused on strengthening clients' motivation for and movement toward their physical activity goals. A pragmatic parallel group randomised controlled trial with 12-month follow-up aims to deliver and assess the effectiveness of the intervention in a format that could realistically be implemented within primary care, workplaces, schools or other similar setting. The study is due to report clinical effectiveness findings in 2014.
\end{abstract}

Keywords

Motivational Interviewing, Social Support, Buddy, Physical Activity

$\mathrm{S}$ ignificant changes in the demographic profile of New Zealand will result in fewer children, more older people and further ageing of the population. Half of New Zealand's population will be 46 years and older by 2051, compared with a median age of 35 years in 2004 (Statistics New Zealand, 2004). For health services, this is significant in two fundamental ways: first, health service utilisation is greatest in the first few and last few years of life; second, these shifts in the demographic profile will also be reflected across the health workforce, potentially resulting in large unsustainable losses of health care professionals. In short, the increasing demand for resources is likely to significantly outstrip the available capacity in the not too distant future. Compounding these demographic factors, the increasing trend in life expectancy in New Zealand is not paralleled by improvements in morbidity, due largely to the progression of non-communicable (lifestyle) diseases, particularly coronary heart disease, obesity and Type 2 diabetes (Ministry of Health, 2001, 2005). Inactive and unfit people have almost double the risk of dying from coronary heart disease compared with more active and fit people (Kohl, Gordon, Villegas, \& Blair, 1992; Lee \& Skerrett, 2001).

Most New Zealanders are exposed to increasingly obesogenic environments and the adverse effects, the so called lifestyle diseases,

\section{${ }^{1}$ Health Sciences Centre, University of Canterbury}

Funding for this study was received from the University of Canterbury Doctoral scholarship programme. The funding body had no role in the study design, collection, analysis and interpretation of data or in the writing of the manuscript. The authors have no conflicts of interest to report.

Correspondence concerning this article should be addressed to: David Brinson, MHealSc, Private Bag 4800, Christchurch, 8020, New Zealand. Email: david.brinson@canterbury.ac.nz. are now obvious. However, engaging in regular, moderately vigorous physical activity can go some way towards offsetting these adverse effects, and the health benefits of regular physical activity are well documented for all age groups (Bouchard \& Shephard, 1994). Early studies conducted by Jeremy Morris and his colleagues (Morris, Heady, Raffle, Roberts, \& Parks, 1953; Morris, Kagan, Pattison, \& Gardner, 1966; Paffenbarger \& Hale, 1975; Paffenbarger, Wing, \& Hyde, 1978) demonstrated the so called independent protective effect of moderately vigorous or vigorous exercise via a series of groundbreaking prospective cohort studies. Moderately vigorous physical activity is positively linked via a cause-and-effect relationship with a range of improved health outcomes (Lee \& Skerrett, 2001) and this relationship is now widely understood and accepted. However, despite the benefits of being more active, most lay-people, researchers, and health professionals would agree that sustained individual-level behaviour change remains very challenging.

\section{Trends in Physical Activity Promotion}

There is growing recognition that health behaviour change is more likely to occur and endure when an individual's environment is supportive of change (McLeroy, Bibeau, Steckler, \& Glanz, 1988). Social-ecological perspectives recognise that society is composed of interconnected elements: individual level, interpersonal, organisational, community, and social, and that these invariably influence one another. Therefore, people who are attempting change are influenced not only by their immediate settings but also by the larger social contexts (both formal and informal) in which these settings are embedded (Brofenbrenner, 1977). There is a growing recognition that it is not particularly helpful to view health problems as residing solely within individuals and quality contemporary health promotion programmes are tending towards a systems approach. A systems approach to physical activity promotion might include 
community-wide campaigns, point-of-decision prompts, school-based programmes, workplace programmes, social support interventions in community settings, enhanced access to places for physical activity, urban design/land-use policies and modification to the built environment (Centers for Disease Control and Prevention, 2008).

Intervention at the population level is important in the overall effort to change sedentary lifestyles. Targeted, well-executed population level campaigns can have small-to-moderate effects not only on health knowledge, beliefs, opinions and attitudes, but also on behaviours as well (Noar 2006). A meta-analysis of health campaign effects on behaviour by Snyder and Hamilton (2002) found effect sizes in the range of 0.17 ( $S D=0.02$ ) for those using a law enforcement message (e.g., seatbelts) to $0.05(\mathrm{SD}=0.04)$ for those not using enforcement messages (e.g., fruit and vegetable consumption, exercise and weight). While the effects might be small for these health promoting behaviours, they are not unimportant because they potentially reach a large number of people and cumulatively, they add up (Glasgow, 2002).

At the individual level, education and brief psychosocial/psychological interventions have been shown to be useful in many areas of health behaviour change, including smoking cessation, changes in nutrition, physical activity and compliance with medication protocols (Burke, Dunn, Atkins, \& Phelps, 2004; Gonder-Frederick, Cox, \& Ritterband, 2002; Pringle, Gilson, Mckenna, \& Cooke, 2009). Notwithstanding the successes, neither population level interventions nor individual level interventions guarantee health behaviour change. For a variety of reasons, programmes often struggle to deal adequately with individual differences in readiness and willingness to change, cultural appropriateness, barriers to equitable access, and myriad other socioeconomic, cognitive and psychological factors (Fuchs, 1998; Ministry of Health, 2002). Health behaviour change remains extremely challenging and change is often not maintained much beyond the intervention period, and there is the persistent tendency for behavioural regression and rebounding (Gonder-Frederick, et al., 2002; McKinlay, 1993). While it is true that modern medicine has evolved to ameliorate many acute illnesses and injuries, it still performs rather less well when faced with the increasing prevalence of lifestyle diseases (Callahan, 2009; Fuchs, 1993, 1998; McKinlay, 1993) and the multi-faceted determinants of health that lie outside of individuals' human biology (Lorig \& Holman, 2003).

Most would agree that a "magic bullet" is unlikely. In attempts to address the particular limitations of both population level and individual level interventions, contemporary perspectives recognise the need for multi-level approaches, sustained over years not months, and the need for multi-sectoral policies to promote physical activity. Such multi-sectoral policies include promoting enabling environments, community involvement, and individual-level intervention (World Health Organization, 2004)

\section{RATIONALE}

This current trial acknowledges recent trends in physical activity promotion and aims to bridge between the individual-level and wider social networks (the inter-personal level) by formally invoking social support via the use of self-selected motivational-buddies. The proposed head-to-head trial has been designed to test a novel adaptation of motivational interviewing (Ml; Miller \& Rollnick, 2002) against usual MI in a physical activity counselling intervention potentially feasible for use in primary care and community settings. The primary outcomes of interest are self-reported physical activity, cardiorespiratory fitness, and healthrelated quality of life (HRQOL). Physical activity reflects the behavioural aims of the intervention and cardiorespiratory fitness reflects the downstream physiological adaptations that may lead to potentially significant health benefits. Also important, HRQOL reflects the psychological aims of the intervention as the HRQOL construct includes the domains roleemotional, vitality, social function, and mental health. The concept of HRQOL acknowledges that people rate their actual situation in relation to their individual expectations.

There is a paucity of evidence for the incremental effectiveness of buddy versus non-buddy interventions in healthcare and this trial aims to add knowledge in this domain. Given the ever present demand for health services and the complex interactions of demand, access, cost and quality, learning how to maximise efficiency in the use of scarce resources is an important research goal.

\section{Why Motivational Interviewing?}

Motivational interviewing (MI) has become a well-recognised style or method of client-centred counselling and the application of $\mathrm{Ml}$ continues to grow at a rapid pace. Only a brief description of $\mathrm{Ml}$ is given here as many other sources provide thorough explanations and descriptions of its application in healthcare and other settings (Arkowitz, 2008; Miller \& Rollnick, 2002, 2009; Miller \& Rose, 2009; Rollnick, Miller, \& Butler, 2008) and the experimental intervention used in this trial is described in detail below. A central tenet of $\mathrm{MI}$ is that the intervention is collaborative in nature and defined by a partnership between the practitioner and the client. Fundamentally, MI involves the activation of peoples' own motivation for change and MI involves a guiding style with the practitioner actively engaged in eliciting the client's intrinsic motivations for change.

There is now considerable evidence (over 200 randomised trials) for the effectiveness of $\mathrm{Ml}$ in the treatment of substance abuse as well as a number of other settings and problem areas, including family practice, chronic care, diabetes, cardiac rehabilitation, oral health (emerging) and diet and exercise. Several systematic reviews and meta-analyses of Ml have now been published (Burke, Arkowitz, \& Menchola, 2003; Hettema, Steele, \& Miller, 2005; Lundahl, Kunz, Brownell, Tollefson, \& Burke 2010; Martins \& McNeil, 2009; Vasilaki, Hosier, \& Cox, 2006) and these generally report positive small-to-medium but clinically significant effects (Abbott \& Freeth, 2008).

A broad range of literature was consulted during the design and refinement of the buddy-MI intervention and in the development of the training resources, including the work of Bandura (1977) on social cognitive theory, Christakis and colleagues (Christakis \& Fowler, 2007) on network effects and health outcomes, Magill, et al. (2010) on motivational interviewing with significant other participation, Moyers and colleagues (Moyers, Martin, Manuel, Miller, \& Ernst, 2007; Moyers, Martin, Manuel, Miller, \& Ernst, 2010) on client language and Miller and Rollnick (2002) and Rollnick et al. (2008) for a general overview of $M$ and its application in health-care settings.

\section{Why a Buddy Intervention?}

The concept of the buddy-system is not new and buddy systems are used formally or informally across a variety of settings ranging from school groups to high hazard workplaces (e.g., search and rescue), the armed forces, business (e.g., mentoring) and healthcare (for example, see May \& West, 2000, for a review of buddy-systems in smoking cessation). Buddy systems generally operate so that two people work together and are able to monitor and help each other, usually for the purpose of orientation or providing support, mentoring, enhancing safety, learning, or motivation, or a combination of these (see also Hurdle, 2001, for a review of social support in health promotion).

While there is no standardised functional definition of a motivational-buddy, in this trial, the buddy role is described as exerting influence in two separate but related domains: the in-session domain 
comprising the structured $\mathrm{Ml}$ part of the programme and the out-ofsession domain, which comprises all other buddy-to-client interactions. Within this framework, the support person or motivational-buddy ideally serves the function of a counselling-buddy (technically a motivationally consistent buddy within the spirit of $\mathrm{Ml}$ ) as well as the more usual emotional/practical support role common to most buddy systems (help with tangible needs, e.g., providing feedback and advice or being an exercise partner or providing other inputs of time and effort or other material resources). Buddies may vary in terms of their enthusiasm, conscientiousness, communication skills, empathy, and availability and generally in the level of support provided. Attempting to positively influence and enhance the supportive relationship between the buddy and the client is therefore another important component of the intervention (see below for more details). However, the goal is not to transform buddies into competent MI therapists, but to guide buddies towards being motivationally consistent in their interactions and on the whole adherent to MI fundamentals: to demonstrate the spirit of MI.

The buddy-intervention aims to bridge between the individual level of intervention and the wider community. Individual level interventions are often resource-limited in their ability to maintain long-term support and they often don't link-in directly with wider social networks and whānau (Māori for "extended family"). The buddy-intervention seeks to address these common limitations by engaging non-health professionals to provide intervention components and ongoing support, with the potential for favourable ripple and inter-personal effects. Consideration has been given to the cultural appropriateness of the intervention, in accordance with the Treaty of Waitangi (New Zealand's founding document) and the focus on partnership is viewed as an important strength.

\section{METHODS}

\section{Design}

Quantitative research methods will be used, based on a pragmatic, parallel group randomised controlled trial (RCT). Blinding the investigator and/or the participants to the treatment received is not possible. Qualitative exit survey data will supplement the findings and provide information on various process outcomes. All procedures were reviewed and approved by the University of Canterbury Human Ethics Committee.

\section{Hypotheses}

The study aim is to investigate the relative effectiveness of $\mathrm{MI}$ delivered in a buddy-system context as compared to usual one-on-one motivational interviewing. The main hypothesis to be tested is that participants in the experimental group will self-report relatively higher levels of physical activity, cardiovascular fitness and health related quality of life at follow-up as compared with control group participants.

\section{Setting}

The study will be conducted in Christchurch, New Zealand, at the University of Canterbury. The University has nearly 19,000 enrolled students, including over 2,000 international students from more than 80 countries and approximately 800 academic staff.

\section{Participants}

Volunteer adults ( $n=60$ ), apparently healthy, relatively physically inactive but able to increase their physical activity. Potential participants will be excluded if in unstable health or if physical activity is contraindicated.

\section{Recruitment and Randomisation}

Participants will be recruited via advertising flyers and other opportunistic recruitment. The study is presented as fundamentally a study of MI with a focus on physical activity and both interventions are presented as real and active therapies. A two-step consent/randomisation strategy is intended to reduce rates of noncompliance and drop-out in the control group by reducing the possibility of resentful demoralisation. Block randomisation will be used via the sealed envelope method (Roberts \& Torgerson, 1998).

\section{Interventions}

\section{Motivational interviewing}

$\mathrm{Ml}$ involves the conscious, disciplined and flexible use of specific communication principles and strategies to evoke a person's own motivations for change. Emphasis is given to the underlying spirit of MI which can be summarised as partnership (an even power relationship and a joint decision making process), autonomy (honouring client autonomy/a detachment from outcome), compassion (unconditional positive regard) and evocation (the process of bringing to mind and harnessing what people already have) (Miller, 2010; Miller \& Rollnick, 2002; Miller \& Rose, 2009). MI involves a number of micro-skills including open questions, affirming, reflecting and summarising (OARS) within an overarching process of engaging, focusing, evoking and planning- and this process can be tailored depending of the needs of the client and the context (Miller, 2010; Miller \& Rollnick, 2002). An MI therapist can also use a range of strategies including agenda-matching, pros and cons, importance and confidence scaling questions, envisioning, rolling with resistance, brainstorming and planning. Another important therapist skill is the ability to resist the righting reflex: the impulse to adopt the expert role and forge ahead of the client in an effort to fix the problem (Miller \& Rollnick, 2002).

Motivational interviewing differs from traditional biomedical counselling with regard to the guiding style of interaction; in addition, the development of discrepancy, supporting self-efficacy, the expression of empathy, empowerment, and encouraging hope and optimism are also components of good Ml practice. MI has the potential to facilitate longterm exercise behaviour change and positively influence peoples' health; however as Miller and Rollnick (2009) point out, "If someone genuinely has no inherent motivation for making a change, MI cannot manufacture it" (p.131).

\section{Buddy-MI}

Motivational Interviewing, as interpreted and adapted here, forms the basis of the proposed buddy-MI intervention model (see Figure 1). In buddy-MI the therapist primarily delivers $\mathrm{Ml}$ but also works with the participant (client) and his/her motivational-buddy to build a therapeutic relationship in which different basic elements of social exchange such as support, reciprocity, accountability and role-modelling may occur and can potentially be channelled to positive effect. Prior to any in-session time, the buddy is provided with background information describing the buddyrole and a range of training resources (as described more fully below). Generally, the focus of the motivational interviewing sessions is on engaging clients and their motivational-buddy in discussions about change, exploring ambivalence about exercise habits, eliciting change talk and commitment language, and planning and discussing how behavioural changes might fit an individual's vision for the future and personal values.

Participants (clients) in the experimental group will be offered faceto-face buddy-MI and follow-up for a period of 12-months and the MI sessions will be conducted with the client's self-selected motivationalbuddy participating. The protocol does not set parameters within which the buddy pair is expected to fit and clients are invited to self-recruit their 


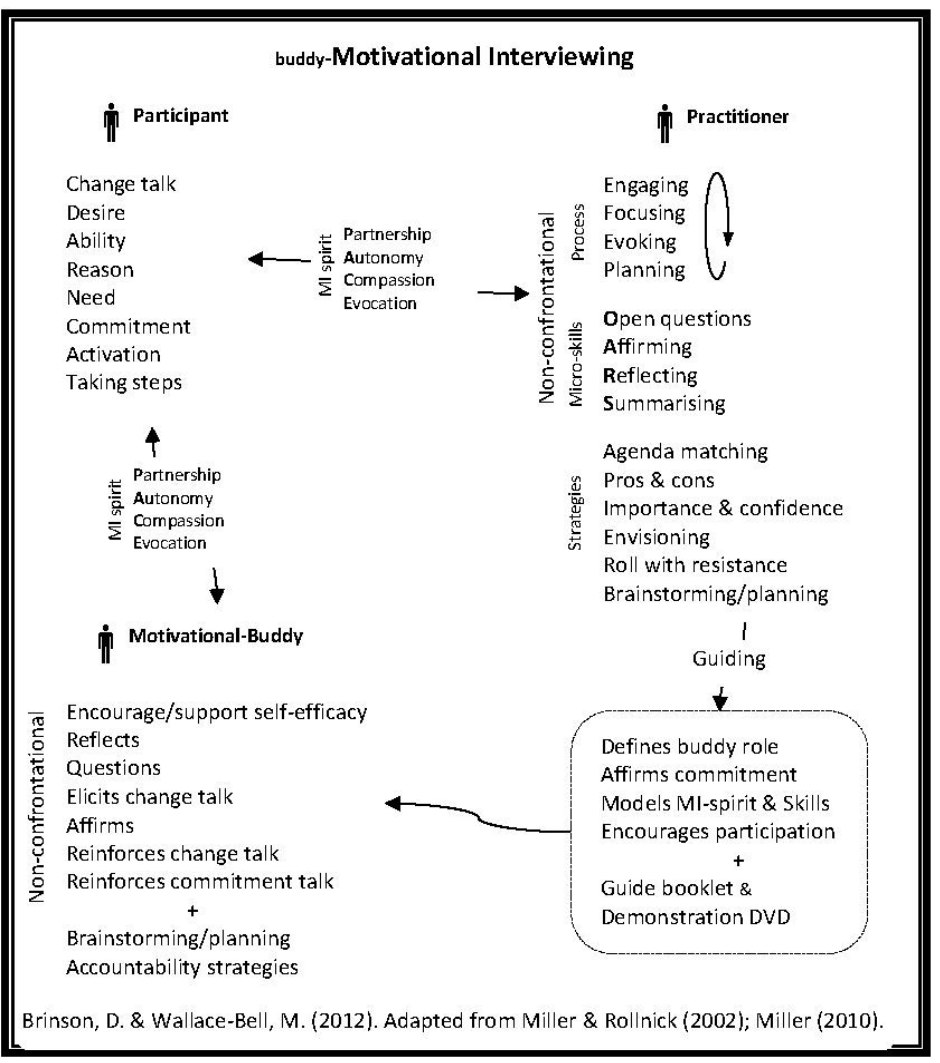

Figure 1

Buddy-MI intervention model.

best choice or best fit buddy. The frequency, timing and duration of the treatment will largely be determined by the participants. Ordinarily, within a 50-minute hour format, it is expected that the intervention will fill a minimum of two sessions ( $<1-2 \mathrm{hrs}$ ) and a maximum of three to five sessions (2-4 hrs) spread over the 12-month intervention period. For all participants, two initial sessions of $\mathrm{Ml}$ will be booked approximately a fortnight apart, but beyond this, the participants will be invited to schedule further sessions to suit their individual needs. Follow-up emails are scheduled for one or two days after each session. These follow-up emails take the form of a personalised note thanking the client/buddy for their participation and confirming the next appointment time. Each followup note also includes one complex reflection and an affirmation relating to a key point from the previous MI session.

Within buddy-MI sessions, the buddy will be encouraged to adopt a non-confrontational communication style, offer reflections on client or therapist statements, question, affirm, support and reinforce change and commitment statements and/or assist with brainstorming and planning. Instruction and guidance in these skills is provided both in the buddy learning package and via in-session modelling by the therapist. The role of the buddy outside of the session time is to be determined entirely by the client-buddy pair (with guidance provided if requested).

The intervention will not follow any specific written therapist manual but as outlined in detail elsewhere (Miller \& Rollnick, 2002), MI can involve a range of standard strategies to elicit change talk including importance and confidence scaling, pros and cons, envisioning and planning for change. Buddy-specific adaptations of these standard MI strategies have been tested for feasibility; pilot study video recordings of client/buddy responses were reviewed and coded with the MISO instrument (Apodaca, Manuel, Moyers, \& Amrhein, 2007) to guide practitioner training. These adaptations generally take the form of asking the buddy to provide an additional perspective on the client or to relay their observations of the client's past challenges, efforts or achievements (often buddies provide these un-prompted). For example, the adaptation of confidence scaling involves asking the buddy to rate his or her perception of the client's ability to take steps towards change (on a scale of 1 to 10). In pilot testing, this more often than not resulted in the buddy scoring the client more highly on the confidence scale and going on to reflect, reinforce, and affirm the client's personal strengths, past achievements and steps already taken towards change. Initial review of pilot session recordings has shown that these buddy-reinforcements and buddy-affirmations commonly elicit client change talk and commitment talk. Eliciting client change talk and commitment talk is generally the objective of using specific strategies in $\mathrm{Ml}$, and in the buddy-MI adaptation, an additional opportunity is created to elicit and reinforce desire, ability, reason, and need statements and to introduce and reinforce positive client attributes.

Agreement between the client and buddy to work on a change plan or to develop an exercise schedule was another common outcome during the pilot interviews: this commitment to planning is commonly initiated collaboratively by the client or buddy rather than by the therapist. Brainstorming and elaborating on the types of out-of-session interactions and the style of communication/accountability that might serve to strengthen the buddy relationship was another common discussion theme. The therapist is thus presented with additional opportunities to reflect, affirm, and selectively reinforce these buddy/client utterances.

Finally, another common theme recorded in the pilot interviews was accountability. Accountability is a component of social engagement that has been used to describe any implied of explicit understanding between two people or any rules and expectations that orient the agent's behaviour (the client) to the role enacted by the overseer (the buddy) (Sharpe, 2000). According to this understanding of accountability, if a client and a buddy establish a relationship based on trust and expected conduct, then a link will be formed between accountability and individual conscience. Client initiated discussions around accountability appear to be common in the buddy-Motivational Interviews and these may exert a motivational influence, although the operationalisation and measurement of accountability and its possible incremental benefits within buddy-MI is beyond the scope of the current research.

\section{Development of buddy-MI training resources}

During the preliminary stages of the buddy-MI pilot, post-session feedback was sought from participating buddies. Buddies typically reported that they were unsure of exactly what their role was and what was expected of them. Attempts to briefly coach buddies in their role and in Ml spirit and micro skills, prior to sessions, proved unsuccessful due to the lack of time to adequately cover the material. As a result of this feedback it became apparent that a more comprehensive approach was required. Further work focused on producing two resources: a guidebook, Buddy basics: Information for motivational-buddies, and a video, Buddy-basics: an instructional video for motivational- buddies.

The information booklet includes introduction and background information and describes the rationale for the study. The content also includes an introduction to the concepts of peer-influence and social networks and their possible effects on health outcomes and an outline of desirable buddy-skills/style along with specific practical examples. The booklet was trialled with buddies and feedback was sought on the content. The booklet was also peer-reviewed by the study supervisors and revisions were made to incorporate all the inputs and to simplify and condense the text.

The instructional DVD was developed in two parts. Part one involved developing a voice-over script and a set of slides and graphics to depict a motivationally adherent communication style, the fundamentals of behaviour change, and the buddy role. Specifics include 
a description of a non-judgmental guiding style, the idea of change vs. status quo, the relevance of personalised goals and values, useful ways to give advice and information (using conditional language) and the importance of avoiding any type of confrontation, directing, arguing or contempt and the importance of being supportive, affirming, and reinforcing of change. The second part of the video involved producing a demonstration role-play of a buddy-MI session. This involved developing a vignette, recruiting actors, recording the session in the studio, audiovisual editing, cover art and post-production. The role-play models some of the different types of positive interactions and buddy-language that might occur during a buddy-MI session and on-screen captions are provided to highlight desirable buddy utterances as they occur. The script of the Buddy basics DVD was developed with reference to the work of Hettema's (2009) MI training videos, findings by Manuel, Houck, and Moyers (2011) in relation to significant other participation in Project MATCH (Project Match Research Group, 1993), and Apodaca and Longabaugh's (2009) review and preliminary evaluation of the mechanisms of change in motivational interviewing. Attempting to quantitatively evaluate the effectiveness of this buddy-training approach is beyond the scope of the present study; however, feedback from buddies following pilot interviews indicated that the materials are helpful.

\section{The active-control intervention}

Because $\mathrm{Ml}$ has been shown to be effective across a range of health promoting behaviours, comparing the experimental buddy-MI to no-treatment would not be overly meaningful, notwithstanding the fact that most people who are sedentary are in all likelihood receiving no treatment. Therefore, the control group will receive an active MI intervention. The control group $\mathrm{Ml}$ intervention differs from the experimental intervention only in that it involves no motivational-buddy.

\section{Treatment delivery}

Two related processes, clinical supervision and fidelity monitoring, are required to ensure that quality $\mathrm{Ml}$ is delivered equivalently to participants in both groups. While related, these two processes are conducted separately as described below.

\section{Therapist skill development / clinical supervision}

The therapist/researcher (the first author) holds a Bachelor of Sports Coaching (BSpC) and a Masters degree in Health Sciences (MHealSc) including sports psychology and MI papers, and a three-day training workshop specific to the MITI 3.1.1 instrument (Moyers, et al., 2010). From this baseline, the therapist/researcher received supervision and feedback spanning the pilot period and ongoing into the main study.

During the pilot period, each video recording was first reviewed by the researcher and scored using the MITI 3.1.1 instrument (Moyers, et al., 2010). The MITI scores were entered into a spreadsheet and graphs were generated to map the following dimensions: Global MI Spirit; Reflection: Question ratio (R:Q); percentage of Open Questions (out of all questions; \%OC); and the percentage of Complex Reflections (out of all reflections; \%CR). In addition, the therapist/researcher carried out self-reflective analysis after selected sessions: writing a reflection (1-2 paragraphs), identifying strengths and less strong characteristics and writing a plan to improve particular aspects of practice as identified.

In addition, the therapist/researcher received fortnightly supervision, feedback and ongoing coaching from a University-based PhD level MI trainer who is a member of the Motivational Interviewing Network of Trainers (MINT) (the second author). Supervision included the review of recordings, coding exercises and calibration of coding, observation and coding of $\mathrm{Ml}$ sessions in real-time and ongoing reviews of performance, with a focus on continuous skill development. A therapist skill level of competency was achieved consistently across all of the MITI subscales and supervision is scheduled for the duration of the study.

\section{Fidelity monitoring}

Ongoing fidelity monitoring will be done via the MITI 3.1.1 instrument (Moyers, et al., 2010) as per the standard recommended protocol for the review of recorded Ml sessions. It is important to note that for the purpose of comparable (between-group) fidelity scoring, therapist utterances that reflect buddy utterances are not counted even if they are directed back to the client. Total therapist utterances (and behaviour counts) may be reduced depending on the level of contribution made by the buddy but the MITI behaviour count ratios hold and the global scores are evaluated using the standard criteria and method. Significant volleys may occur between the buddy and the client but these are not captured by the MITI. Both the Motivational Interviewing Skill Code (MISC) (Miller, Moyers, Ernst, \& Amrhein, 2008) and the Motivational Interviewing with Significant Others (MISO) (Apodaca, et al., 2007) could be applied to analyse buddy utterances and provide addition data but this is beyond the scope of the current study.

The fidelity monitoring schedule will be based on retrospective, random, single blinded sampling of $25 \%$ of all interviews per quarter. The randomly selected 20 min video clips will be collated onto one DVD for review and rating by the study supervisor. Fidelity data (in particular between-group comparisons) will be analysed and fed back to the therapist during supervision and subsequently used in later data analyses. Table 1 shows the pilot study fidelity scores based on 16 firstsession interviews, indicating provision of $\mathrm{Ml}$ above competency benchmarks. Similar data will be produced for the duration of the main study.

Table 1.

Pilot study fidelity scores via the MITI 3.1.1 instrument, $n=16$

\begin{tabular}{ccc}
\hline Measure & Control group & Experimental group \\
\hline Global clinician rating & 4.45 & 4.13 \\
Reflection to Question Ratio (R:Q) & 2.2 & 2.1 \\
Percent Open Questions (\%OC) & $76 \%$ & $78 \%$ \\
Percent Complex Reflections (\%CR) & $73 \%$ & $86 \%$ \\
Percent MI-Adherent (\% MIA) & $100 \%$ & $100 \%$ \\
\hline
\end{tabular}

\section{Outcome Measures}

Outcome data will be collected in several different ways: self-report via on-line multi-choice questionnaires, objective self-administered fitness tests, coding of video-recorded Ml sessions, and free-text exit interview responses. A process evaluation will explore the implementation of the intervention, including number of sessions, treatment fidelity, and participant adherence to the assessment protocol and will include exit survey information describing the participants' own experience of being part of the trial. Data from exit interviews will be analysed for emergent themes using NVIVO ${ }^{\text {TM }}$ software. (See Table 2 for detailed information on the study measures.)

\section{Statistical Methods}

All statistical analyses will be overseen by the UC Health Sciences statistician/advisor to ensure that appropriate and robust procedures are followed. The SPSS ${ }^{\mathrm{TM}}$ software will be used for the analysis. The intention-to-treat principle will be adhered to such that all randomised 
Table 2.

Outcome Measures

\begin{tabular}{|c|c|c|c|}
\hline Outcome measure & Instrument & Explanation & Administered \\
\hline \multicolumn{4}{|l|}{ Primary } \\
\hline Self-reported physical activity & $\begin{array}{l}\text { International Physical Activity Questionnaire (IPAQ) } \\
\text { (Craig et al., 2003) }\end{array}$ & $\begin{array}{l}\text { Long form - last 7-days recall, self- } \\
\text { administered on-line questionnaire }\end{array}$ & Baseline, 1, 3 \& 12-months \\
\hline Cardiorespiratory fitness & Cooper 12-minute run test (Cooper, 1968) & $\begin{array}{l}\text { Sub-maximal running/walking test to } \\
\text { assess aerobic fitness: converted to } \\
\mathrm{VO}_{2 \mathrm{Max}} \text { as per Cooper (1968) }\end{array}$ & Baseline, 1, 3 \& 12-months \\
\hline Health-related quality of life & SF36v2 (Quality Metric, USA) & $\begin{array}{l}\text { Self-administered short-form health- } \\
\text { related quality of life survey }\end{array}$ & Baseline, 1, 3 \& 12-months \\
\hline \multicolumn{4}{|l|}{ Secondary } \\
\hline $\begin{array}{l}\text { Exercise readiness (stage of } \\
\text { change) }\end{array}$ & $\begin{array}{l}\text { Exercise Stages of Change - Short Form (Marcus, } \\
\text { Selby, Niaura, \& Rossi, 1992) }\end{array}$ & $\begin{array}{l}\text { One item short form exercise } \\
\text { readiness questionnaire based on } \\
\text { the Transtheoretical Model } \\
\text { (Prochaska \& DiClemente, 1983) }\end{array}$ & Baseline, 1, 3 \& 12-months \\
\hline Self-efficacy & $\begin{array}{l}\text { Generalised Self-Efficacy scale (GSE) (Schwarzer } \\
\text { et al., 1981) with additional Exercise Self-efficacy } \\
\text { Scale (ESE) items added (Schwarzer \& Renner, } \\
\text { 2000) }\end{array}$ & $\begin{array}{l}\text { Self-reported perceived self-efficacy } \\
\text { and exercise specific self-efficacy }\end{array}$ & Baseline, 1, 3 \& 12-months \\
\hline Social support & $\begin{array}{l}\text { Norbeck Social Support Questionnaire (NSSQ) } \\
\text { (Norbeck, Lindsey, \& Carrieri, 1981, 1983) }\end{array}$ & $\begin{array}{l}\text { Measures multiple components of } \\
\text { social support including functional } \\
\text { properties, network properties, } \\
\text { amount of support from specific } \\
\text { sources as well descriptive data } \\
\text { about recent losses }\end{array}$ & Baseline \& 12-months \\
\hline $\begin{array}{l}\text { Satisfaction with the social } \\
\text { relationship } \\
\text { group only) }\end{array}$ & $\begin{array}{l}\text { Partner Interaction Questionnaire (PIQ-20) (Cohen } \\
\text { \& Lichtenstein, 1990) }\end{array}$ & $\begin{array}{l}\text { The PIQ- } 20 \text { modified to change the } \\
\text { context from smoking cessation to } \\
\text { physical activity }\end{array}$ & 12-months \\
\hline $\begin{array}{l}\text { Motivational-buddy empathy / } \\
\text { helping style (experimental } \\
\text { group only) }\end{array}$ & $\begin{array}{l}\text { The Helpful Responses Questionnaire (HRQ) } \\
\text { (Miller, Hedrick, \& Orlofsky, 1991) }\end{array}$ & $\begin{array}{l}\text { A measure of helping-style/ empathy, } \\
\text { a brief free-response questionnaire }\end{array}$ & Baseline \\
\hline \multicolumn{4}{|l|}{ Ml outcomes } \\
\hline Treatment fidelity & $\begin{array}{l}\text { Motivational Interviewing Treatment Integrity } \\
\text { instrument (MITI 3.1.1) (Moyers, et al., 2010) }\end{array}$ & $\begin{array}{l}\text { Used to code and rate randomly } \\
\text { selected interview recordings }\end{array}$ & $\begin{array}{l}25 \% \text { random selection of all Ml } \\
\text { session recordings }\end{array}$ \\
\hline \multicolumn{4}{|l|}{ Qualitative } \\
\hline Participant/Buddy exit surveys & A brief six question free-response questionnaire & Analysed using thematic analysis & 12-months \\
\hline
\end{tabular}

participants will be analysed in the groups to which they were originally assigned, regardless of their adherence and the treatment they actually receive and regardless of subsequent dropout or any other deviation from the protocol (Moher, Schulz, \& Altman, 2001). If a total of 60 participants enter this two-treatment parallel-design study, the probability is 80 percent that the study will detect a treatment difference of 0.66 $\mathrm{kcal} / \mathrm{kg} /$ day (approximately 10 minutes of moderate-intensity physical activity/day) at a two-sided 0.05 significance level. Participants' baseline characteristics will be analysed, intervention dose-by-group will be calculated, and treatment fidelity data will be analysed. Statistical 
adjustment will be made in the case of any significant between-group differences.

Between-group changes in means across the primary outcomes will be analysed. Multivariable analysis will be applied to adjust for the possible influence of confounding variables including age, gender and ethnicity. Logistic regression analysis will be used to examine physical activity levels in relation to current recommendations. Cox proportional hazards regression will be used to model participants' progression in relation to the Cooper Institute's fitness categories (Cooper, 1968). Between-group differences in $\mathrm{HRQOL}$ will be investigated using analysis of covariance (ANCOVA). Differences in mean scores across the primary outcomes will be compared with previously published estimates of clinically important differences (CIDs) for the primary outcomes.

\section{DISCUSSION}

The study, due to report its findings in 2014, aims to test the incremental effectiveness of motivational-buddy support in addition to one-on-one motivational interviewing in people who have expressed an interest in becoming more physically active. It uses a novel intervention design incorporating client-selected motivational-buddies in an effort to mitigate the twin problems of poor adherence and behavioural regression that are commonly associated with physical activity promotion programmes. Strengths of the study include the use of a pragmatic RCT design in a realistic setting, relatively unrestricted entry criteria and analysis of the primary outcomes in accordance with an intention to treat protocol. Together these features will help to provide information about the potential impact of the intervention when introduced into a service, as compared to the efficacy information typically provided by more controlled clinical trials.

As well as the effectiveness data, the study also aims to provide qualitative information on the implementation of the intervention (structure/design/dynamics of the buddy-MI sessions) that may be helpful in the refinement of future buddy-MI iterations. The buddy-MI intervention's therapeutic effectiveness is yet to be demonstrated but the potential implications for the health-care system and the wider community are reduced resource utilisation and healthier lifestyles.

\section{REFERENCES}

Abbott, S., \& Freeth, D. (2008). Social capital and health: Starting to make sense of the role of generalized trust and reciprocity. Journal of Health Psychology, 13(7), 874-883, from

http://search.ebscohost.com/login. aspx?direct=true\&db=psyh\&AN=2008$\underline{14124-005 \& \text { site }=\text { ehost-live }}$

Apodaca, T., \& Longabaugh, R. (2009). Mechanisms of change in motivational interviewing: A review and preliminary evaluation of the evidence. Addiction, 104(5), 705-715, from

http://www.ncbi.nlm.nih.gov/pmc/articles/PMC2756738/pdf/nihms114387.pdf

Apodaca, T., Manuel, J. K., Moyers, T. B., \& Amrhein, P. (2007). Motivational Interviewing with Significant Others (MISO) Coding Manual.Unpublished manuscript.

Arkowitz, H. (2008). Motivational interviewing in the treatment of psychological problems. New York: Guilford Press.

Bandura, A. (1977). Self-efficacy: Toward a unifying theory of behavioral change. Psychological Review(84), 191-215, from http://www.ou.edu/cls/online/lstd5423/pdfs/bandura.pdf

Bouchard, C., \& Shephard, R. J. (1994). Physical activity, fitness, and health: the model and key concepts. In C. Bouchard, R. J. Shephard \& T. Stephens
(Eds.), Physical activity, fitness and health (pp. 77-88). Champaign, IL: Human Kinetics.

Brofenbrenner, U. (1977). Toward an Experimental Ecology of Human Development. American Psychologist, 32(7), 513-531, from http://maft.dept.uncg.edu/hdf/facultystaff/Tudge/Bronfenbrenner\%201977.pdf

Burke, B. L., Arkowitz, H., \& Menchola, M. (2003). The efficacy of motivational interviewing: A meta-analysis of controlled clinical trials, Journal of Consulting and Clinical Psychology (Vol. 71, pp. 843-861).

Burke, B. L., Dunn, C. W., Atkins, D. C., \& Phelps, J. S. (2004). The emerging evidence base for motivational interviewing: A meta-analytic and qualitative inquiry. Journal of Cognitive Psychotherapy, 18(4), 309-322, from http://faculty.fortlewis.edu/burke b/Teaching\%20Portfolio/JCP-Burke04.pdf

Callahan, D. (2009). Taming the beloved beast : how medical technology costs are destroying our health care system. Princeton: Princeton University Press.

Centers for Disease Control and Prevention (2008). State Nutrition, Physical Activity and Obesity (NPAO) Program: Technical Assistance Manual: Division of Nutrition, Physical Activity and Obesity.

Christakis, N. A., \& Fowler, J. H. (2007). The Spread of Obesity in a Large Social Network over 32 Years. New England Journal of Medicine, 357(4), 370-379, from http://www.nejm.org/doi/abs/10.1056/NEJMsa066082

Cohen, S., \& Lichtenstein, E. (1990). Partner behaviors that support quitting smoking. J Consult Clin Psychol, 58(3), 304-309, from http://www.psy.cmu.edu/ scohen/smoking90.pdf

Cooper, K. H. (1968). A Means of Assessing Maximal Oxygen Intake: Correlation Between Field and Treadmill Testing. JAMA, 203(3), 201-204, from http://jama.ama-assn.org/cgi/content/abstract/203/3/201

Fuchs, V. R. (1993). The future of health policy / Victor R. Fuchs. Cambridge, Mass. : Harvard University Press.

Fuchs, V. R. (1998). Who shall live? : health, economics, and social choice / Victor R. Fuchs (Expanded ed. ed.). Singapore ; River Edge, NJ: World Scientific.

Glasgow, R. E. (2002). Evaluation of theory-based interventions: The RE-AIM model. In K. Glanz, F. M. Lewis \& B. K. Rimer (Eds.), Health behavior and health education: Theory, research, and practice (3 ed., pp. 531-544). San Francisco, CA: John Wiley \& Sons.

Gonder-Frederick, L. A., Cox, D. J., \& Ritterband, L. M. (2002). Diabetes and behavioral medicine: the second decade. J Consult Clin Psychol, 70(3), 611625, from http://umassmed.edu/uploadedFiles/diabetes/resources/DiabetesBehavioralM edicine.pdf

Hettema, J. (2009). Motivational Interviewing training video: a tool for learners, Disc 1 [DVD]: Land of Enchantment Productions.

Hettema, J., Steele, J., \& Miller, W. R. (2005). Motivational interviewing, Annual Review of Clinical Psychology (Vol. 1, pp. 91-111 Downloaded from: http://www.annualreviews.org/doi/pdf/110.1146/annurev.clinpsy.1141.102803. 143833).

Hurdle, D. E. (2001). Social support: a critical factor in women's health and health promotion. Health \& Social Work, 26(2), 72-79, from http://search.ebscohost.com/login.aspx?direct=true\&db=c8h\&AN=200109367 $\underline{1 \& \text { site }=\text { ehost-live }}$

Kohl, H. W., Gordon, N. F., Villegas, J. A., \& Blair, S. N. (1992). Cardiorespiratory fitness, glycemic status, and mortality risk in men. Diabetes Care, 15(2), 184192, from http://care.diabetesjournals.org/content/15/2/184.full.pdf+html

Lee, I. M., \& Skerrett, P. J. (2001). Physical activity and all-cause mortality: what is the dose-response relation? Med Sci Sports Exerc, 33(6 Suppl), S459-471; 
discussion S493-454, from

http://www.indiana.edu/ k562/articles/role/Lee\%20PA\%20morbidity.pdf

Lorig, K. R., \& Holman, H. (2003). Self-management education: history, definition, outcomes, and mechanisms. Ann Behav Med, 26(1), 1-7, from http://www.cofammedresidencies.org/PCMH\%20Materials/Self\%20Mgmt $\% 20$ \%20K\%20Lorig\%20\%20\%202-04\%20(2).Shepherd.pdf

Lundahl, B. W., Kunz, C., Brownell, C., Tollefson, D., \& Burke, B. L. (2010). A MetaAnalysis of Motivational Interviewing: Twenty-Five Years of Empirical Studies. Research on Social Work Practice, 20(2), 137-160, from http://rsw.sagepub.com/content/20/2/137.abstract

Magill, M., Mastroleo, N. R., Apodaca, T., Barnett, N. P., Colby, S. M., \& Monti, P. M. (2010). Motivational interviewing with significant other participation: Assessing therapeutic alliance and patient satisfaction and engagement. [doi: DOI: 10.1016/j.jsat.2010.07.006]. Journal of Substance Abuse Treatment, 39(4), 391-398, from http://www.sciencedirect.com/science/article/B6T9050XTT2D-3/2/0864a5c78b5197eafe976fc0ac6cda4b

Manuel, J. K., Houck, J. M., \& Moyers, T. B. (2011). The Impact of Significant Others in Motivational Enhancement Therapy: Findings from Project MATCH. Behavioural and Cognitive Psychotherapy, FirstView, 1-16, from http://dx.doi.org/10.1017/S1352465811000592

Marcus, B. H., Selby, V. C., Niaura, R. S., \& Rossi, J. S. (1992). Self-efficacy and the stages of exercise behavior change. Research Quarterly for Exercise and Sport, 63, 60-66, from http://www.ncbi.nlm.nih.gov/pubmed/1574662

Martins, R. K., \& McNeil, D. W. (2009). Review of Motivational Interviewing in promoting health behaviors. Clinical Psychology Review, 29(4), 283-293, from http://ac.els-cdn.com/S0272735809000099/1-s2.0S0272735809000099main.pdf? tid=f1409ea0c077ceca07d80ccd6a9ef6ed\&acdnat=1334794230 6c47e9a91b052af2394592a860772b4f

May, S., \& West, R. (2000). Do social support interventions ("buddy systems") aid smoking cessation? A review. Tob Control, 9(4), 415-422, from http://bmjtobacco.highwire.org/content/9/4/415.full.pdf+html

McKinlay, J. B. (1993). The promotion of health through planned sociopolitical change: challenges for research and policy. Soc Sci Med, 36(2), 109-117, from http://www.ncbi.nlm.nih.gov/pubmed/8421787

McLeroy, K. R., Bibeau, D., Steckler, A., \& Glanz, K. (1988). An ecological perspective on health promotion programs. Health Educ Q, 15(4), 351-377, from http://www.uib.no/filearchive/filetopic paper-ecological-perspectivemcleroy.pdf

Miller, W. R. (2010). Looking Forward to MI-3: A work in progress. Forum plenary, MINT Forum, October 2010.

Miller, W. R., Hedrick, K. E., \& Orlofsky, D. R. (1991). The Helpful Responses Questionnaire: a procedure for measuring therapeutic empathy. Journal of Clinical Psychology, 47(3), 444-448, from http://search.ebscohost.com/login.aspx?direct=true \&db=mnh\&AN=2066417\& site=ehost-live

Miller, W. R., Moyers, T. B., Ernst, D., \& Amrhein, P. (2008). Manual for the Motivational Interviewing Skill Code (MISC) Version 2.1. Retrieved from http://casaa.unm.edu/download/misc.pdf

Miller, W. R., \& Rollnick, S. (2002). Motivational interviewing : preparing people for change (2nd ed.). New York ;: London : Guilford Press.

Miller, W. R., \& Rollnick, S. (2009). Ten things that motivational interviewing is not. Behav Cogn Psychother, 37(2), 129-140, from http://ihatetherapy.com/wpcontent/uploads/2010/07/miller-09-pdf1.pdf
Miller, W. R., \& Rose, G. S. (2009). Toward a Theory of Motivational Interviewing. Am Psychol, 64(6), 527-537, from http://www.ncbi.nlm.nih.gov/pmc/articles/PMC2759607/pdf/nihms146933.pdf

Ministry of Health (2001). The burden of disease and injury in New Zealand. Public Health Intelligence Occasional Bulletin No. 1. Retrieved from http://www.moh.govt.nz/notebook/nbbooks.nsf/0/E916C53D599AE126CC256 9F0007AAA23/\$file/BurdenofDisease.pdf

Ministry of Health (2002). Reducing inequalities in health. Retrieved from http://www.maorihealth.govt.nz/moh.nsf/pagesma/307/\$File/Reduclnegal.pdf

Ministry of Health (2005). An indication of New Zealanders' health 2004. Retrieved from

http://www.fmhs.auckland.ac.nz/faculty/tkhm/tumuaki/ docs/An Indication of NZers Health.pdf

Moher, D., Schulz, K. F., \& Altman, D. G. (2001). The CONSORT statement: revised recommendations for improving the quality of reports of parallel-group randomised trials. [doi: DOI: 10.1016/S0140-6736(00)04337-3]. The Lancet, 357(9263), 1191-1194, from

http://www.sciencedirect.com/science/article/B6T1B-42VM8B615/2/228de7def635514151bd912110110289

Morris, J. N., Heady, J. A., Raffle, P. A., Roberts, C. G., \& Parks, J. W. (1953). Coronary heart-disease and physical activity of work. Lancet, 265, 10531057, from http://www.ncbi.nlm.nih.gov/pmc/articles/PMC2027542/pdf/brmedj030820009.pdf

Morris, J. N., Kagan, A., Pattison, D. C., \& Gardner, M. J. (1966). Incidence and prediction of ischaemic heart-disease in London busmen. Lancet, 2(7463), 553-559, from http://www.ncbi.nlm.nih.gov/pubmed/4161611

Moyers, T. B., Martin, T., Manuel, J. K., Miller, W. R., \& Ernst, D. (2007). Revised global scales: Motivational interviewing treatment integrity 3.0. from http://casaa.unm.edu/download/miti3.pdf

Moyers, T. B., Martin, T., Manuel, J. K., Miller, W. R., \& Ernst, D. (2010). Revised Global Scales: Motivational Interviewing Treatment Integrity 3.1.1. Retrieved from http://casaa.unm.edu/download/miti3 1.pdf

Norbeck, J. S., Lindsey, A. M., \& Carrieri, V. L. (1981). The development of an instrument to measure social support. Nurs Res, 30(5), 264-269, from http://www.ncbi.nlm.nih.gov/pubmed/7027185

Norbeck, J. S., Lindsey, A. M., \& Carrieri, V. L. (1983). Further development of the Norbeck Social Support Questionnaire: normative data and validity testing. Nurs Res, 32(1), 4-9, from http://psycnet.apa.org/psycinfo/1983-27052-001

Paffenbarger, R. S., \& Hale, W. E. (1975). Work activity and coronary heart mortality. N Engl J Med, 292(11), 545-550, from http://www.neim.org/doi/full/10.1056/NEJM197503132921101

Paffenbarger, R. S., Wing, A. L., \& Hyde, R. T. (1978). Physical activity as an index of heart attack risk in college alumni. Am J Epidemiol, 108(3), 161-175, from http://www.ncbi.nlm.nih.gov/pubmed/707484

Pringle, A., Gilson, N., Mckenna, J., \& Cooke, C. (2009). An Evaluation of the Local Exercise Action Pilots and Impact on Moderate Physical Activity. Health Education Journal, 68(3), 179-185,

Prochaska, J. O., \& DiClemente, C. C. (1983). Stages and processes of selfchange of smoking: toward an integrative model of change. J Consult Clin Psychol, 51(3), 390-395, from http://www.sfu.ca/medialab/archive/2010/426/Readings/Theoretical\%20Framework/Stages\%20of\%20 Change\%20-\%20Prochaska\%201983.pdf

Project Match Research Group (1993). Project MATCH: Rationale and Methods for a Multisite Clinical Trial Matching Patients to Alcoholism Treatment. 
Alcoholism: Clinical and Experimental Research, 17(6), 1130-1145, from http://dx.doi.org/10.1111/i.1530-0277.1993.tb05219.x

Roberts, C., \& Torgerson, D. (1998). Understanding controlled trials: Randomisation methods in controlled trials. BMJ, 317(7168), 1301-1310, from http://www.bmj.com

Rollnick, S., Miller, W. R., \& Butler, C. (2008). Motivational interviewing in health care : helping patients change behavior. New York: Guilford Press.

Sharpe, V. A. (2000). Behind Closed Doors: Accountability and Responsibility in Patient Care. Journal of Medicine and Philosophy: A Forum for Bioethics and Philosophy of Medicine, 25(1), 28 - 47, from http://www.informaworld.com/10.1076/0360-5310(200002)25:1;1-V;FT028

Snyder, L. B., \& Hamilton, M. A. (2002). A meta-analysis of U.S. health campaign effects on behavior: emphasize enforcement, exposure, and new information, and beware the secular trend. In R. C. Hornik (Ed.), Public health communication: evidence for behavior change. Mahwah, NJ.: USA: Lawrence Erlbaum Associates, Inc.

Statistics New Zealand (2004). National Population Projections 2004(base) - 2051. from

http://www.stats.govt.nz/browse for stats/population/estimates and projecti ons/NationalPopulationProjections HOTP04-51.aspx.

Vasilaki, E. I., Hosier, S. G., \& Cox, W. M. (2006). The efficacy of motivational interviewing as a brief intervention for excessive drinking: a meta-analytic review. Alcohol and Alcoholism, 41(3), 328-335, from http://alcalc.oxfordjournals.org/content/41/3/328.abstract

World Health Organization (2004). Global Strategy on Diet, Physical Activity and Health. Geneva: World Health Organization.

This work is licensed under a Creative Commons AttributionNoncommercial-No Derivative Works 3.0 United States License.

This journal is published by the University Library System of the University of Pittsburgh as part of its D-Scribe Digital Publishing Program, and is cosponsored by the Motivational Interviewing Network of Trainers. 